\title{
Deciphering Nd and Sm isotope composition in chondrites
}

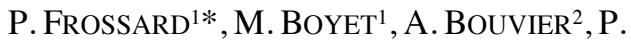 \\ BONNAND $^{1}$, D. AUCLAIR ${ }^{1}$ \\ ${ }^{1}$ Université Clermont Auvergne, CNRS, IRD, OPGC, \\ Laboratoire Magmas et Volcans, Clermont-Ferrand, \\ France \\ ${ }^{2}$ Bayerisches Geoinstitut, Universität Bayreuth, Germany \\ (*correspondence: paul.frossard@uca.fr)
}

A mass-independent isotope dichotomy has been identified between non-carbonaceous (NC) and carbonaceous (CC) meteorites for many elements (e.g. O, Cr, Ti, Mo, Ru) [1]. These isotopic anomalies can be used as tracers to better constrain the bulk composition of the Earth. The origin of this dichotomy between chondrite groups is still debated but the incorporation of CAI in CC appear to play an important role for some isotopic ratios such as ${ }^{46} \mathrm{Ti} /{ }^{47} \mathrm{Ti}[2]$ and ${ }^{144} \mathrm{Sm} /{ }^{152} \mathrm{Sm}$ [3] but not other isotopic variations.

In order to determine the origin of variation in the bulk chondrite isotope composition for both $\mathrm{Sm}$ and $\mathrm{Nd}$, we analysed sequential acid leachates and residues for two enstatite chondrites (EL3 and EH3), one ordinary chondrite (L3.2), as well as whole-rock CC. Chromium isotope composition of these fractions will also be measured. The recent development of $10^{13} \Omega$ resistors allows us to measure $\mathrm{Sm}$ isotope composition of small fractions of leachates. The small resolved anomalies in the ordinary chondrite leachates and residue reflect the destruction of presolar grains by metamorphism despite a low petrological type. The two enstatite chondrites display small anomalies in the mildest leachates complementary with large anomalies in the residues. The variation in $\mathrm{Nd}$ isotope composition of leachates in chondrites is best explained by mixing of non$\mathrm{SiC}$ matter and presolar $\mathrm{SiC}$ grains. Samarium isotope composition of leachates in chondrites is more complex as the ${ }^{144} \mathrm{Sm} /{ }^{152} \mathrm{Sm}$ is very sensitive to CAI-like components, depleted in p-process. These new data allow us to constrain the s-process signature for $\mathrm{Sm}$ isotopes that is currently not well understood. The difference in $\mathrm{Sm}$ and $\mathrm{Nd}$ isotope compositions in bulk carbonaceous and non-carbonaceous chondrites appears not to be influenced by CAIs, with the exception of ${ }^{144} \mathrm{Sm}$, but follows the s-process mixing trend. We will explore the relative contribution of $\mathrm{p}$ - and s-process in the ${ }^{142} \mathrm{Nd} /{ }^{144} \mathrm{Nd}$ composition of the chondrite groups in more details to give further constraints on Earth's ${ }^{142} \mathrm{Nd} /{ }^{144} \mathrm{Nd}$.

[1] Warren et al., 2011, EPSL. [2] Burkhardt et al., 2019, GCA. [3] Bouvier and Boyet, 2016, Nature. 